Elsevier required licence: (C) <2019>. This manuscript version is made available under the CC-BY-NC-ND 4.0 license http://creativecommons.org/ licenses/by-nc-nd/4.0/. The definitive publisher version is available online at [insert DOI] 
Elsevier Editorial System(tm) for Journal of Computational and Applied Mathematics

Manuscript Draft

Manuscript Number: CAM-D-18-00226

Title: Numerical mode matching for sound propagation in silencers with granular material

Article Type: VSI:Conference IMM_2017

Section/Category: VSI:Conference IMM_2017

Keywords: Sound Attenuation; Silencer; Granular Material; Numerical Mode Matching; Finite Element Method; Computational Performance.

Corresponding Author: Dr. Francisco David Denia, Senior Lecturer, Ph.D.

Corresponding Author's Institution: Universitat Politècnica de València

First Author: Eva M Sánchez-Orgaz, Senior Researcher, Ph.D.

Order of Authors: Eva M Sánchez-Orgaz, Senior Researcher, Ph.D.;

Francisco David Denia, Senior Lecturer, Ph.D.; Luis Baeza, Professor, Ph.D.; Ray Kirby, Senior Lecturer, Ph.D.

Abstract: This work presents an efficient numerical approach based on the combination of the mode matching technique and the finite element method (FEM) to model the sound propagation in silencers containing granular material and to evaluate their acoustic performance through the computation of transmission loss (TL). The methodology takes into account the presence of three-dimensional (3D) waves and the corresponding higher order modes, while reducing the computational expenditure of a full 3D FE calculation. First, the wavenumbers and transversal pressure modes associated with the silencer cross section are obtained by means of a two-dimensional FE eigenvalue problem, which allows the consideration of arbitrary transversal geometries and material heterogeneities. The numerical approach considers the possibility of using different filling levels of granular material, giving rise to cross sections with abrupt changes of properties located not only in the usual central perforated passage, but also in the transition between air and material, that involves a significant change in porosity. After solving the eigenvalue problem, the acoustic fields (acoustic pressure and axial velocity) are coupled at geometric discontinuities between ducts through the compatibility conditions to obtain the complete solution of the wave equation and the acoustic performance (TL). The granular material is analysed as a potential alternative to the traditional dissipative silencers incorporating fibrous absorbent materials. Sound propagation in granular materials can be modelled through acoustic equivalent properties, such as complex and frequency dependent density and speed of sound. TL results computed by means of the numerical approach proposed here show good agreement with full 3D FE calculations and experimental measurements. As expected, the numerical mode matching outperforms the computational expenditure of the full 3D FE approach. Different configurations have been studied to determine the influence on the TL of several parameters such as the size of the material grains, the filling 
level of the chamber, the granular material porosity and the geometry of the silencer cross section. 


\section{HIGHLIGHTS}

A numerical mode matching approach is proposed to model sound propagation in silencers containing granular material.

It allows efficient computation of arbitrary transversal geometries, material heterogeneities and abrupt changes of properties.

TL curves computed through the proposed approach agree with full 3D FE calculations and experimental measurements.

The acoustic influence of several parameters is analyzed, including granular size and geometry of the silencer cross section. 


\title{
Numerical mode matching for sound propagation in silencers with granular material
}

\author{
E. M. Sánchez-Orgaz , F. D. Denia ${ }^{\mathrm{a}, *}$, L. Baeza $^{\mathrm{b}}$, R. Kirbyc
}

${ }^{a}$ Centro de Investigación en Ingeniería Mecánica, Universitat Politècnica de València, Camino de Vera $s / n, 46022$ Valencia, Spain

bInstitute of Sound and Vibration Research, University of Southampton, Southampton, SO17 1BJ, United Kingdom

'Centre for Audio, Acoustics and Vibration, University of Technology, Sydney, NSW 2007, Australia

\section{${ }^{*}$ Corresponding author:}

Dr. F. D. Denia

Centro de Investigación en Ingeniería Mecánica

Universitat Politècnica de València

Camino de Vera s/n

46022 Valencia

Spain

Tel: +34 963877007 Ext: 76225

Fax: +3496387 7629

e-mail: fdenia@mcm.upv.es 


\section{ABSTRACT}

This work presents an efficient numerical approach based on the combination of the mode matching technique and the finite element method (FEM) to model the sound propagation in silencers containing granular material and to evaluate their acoustic performance through the computation of transmission loss (TL). The methodology takes into account the presence of three-dimensional (3D) waves and the corresponding higher order modes, while reducing the computational expenditure of a full 3D FE calculation. First, the wavenumbers and transversal pressure modes associated with the silencer cross section are obtained by means of a two-dimensional FE eigenvalue problem, which allows the consideration of arbitrary transversal geometries and material heterogeneities. The numerical approach considers the possibility of using different filling levels of granular material, giving rise to cross sections with abrupt changes of properties located not only in the usual central perforated passage, but also in the transition between air and material, that involves a significant change in porosity. After solving the eigenvalue problem, the acoustic fields (acoustic pressure and axial velocity) are coupled at geometric discontinuities between ducts through the compatibility conditions to obtain the complete solution of the wave equation and the acoustic performance $(T L)$. The granular material is analysed as a potential alternative to the traditional dissipative silencers incorporating fibrous absorbent materials. Sound propagation in granular materials can be modelled through acoustic equivalent properties, such as complex and frequency dependent density and speed of sound. TL results computed by means of the numerical approach proposed here show good agreement with full 3D FE calculations and experimental measurements. As expected, the numerical mode matching outperforms the computational expenditure of the full 3D FE approach. Different configurations have been studied to determine the influence on the $T L$ of several parameters such as the size of the material grains, the filling level of the chamber, the granular material porosity and the geometry of the silencer cross section.

Keywords: Sound Attenuation; Silencer; Granular Material; Numerical Mode Matching; Finite Element Method; Computational Performance 


\section{INTRODUCTION}

Multidimensional methods are widely used for the acoustic modelling of silencers and other exhaust devices [1]. Accurate predictions of the sound attenuation performance at mid and high frequencies require the consideration of threedimensional waves corresponding to higher order modes. Numerical approaches such as the boundary element method (BEM) and the finite element method (FEM) are usual design tools, although the associated computational expenditure of these fully numerical schemes can be considerable as the number of degrees of freedom increases [2, 3]. Thus, an effort has been made in the last two decades with a view to developing alternative modelling techniques that provide improved computational efficiency without sacrificing accuracy. Some of these techniques are based on hybrid approaches [4-11] that combine analytical and numerical aspects of the wave propagation. For example, in silencers with irregular but axially uniform cross section, a numerical approach can be used to model the transversal governing eigenequation [7]. The complete solution of the acoustic field in a particular silencer subdomain is obtained by considering the contribution of the axial propagating terms analytically. Finally, the acoustic coupling of all the subdomains involved is achieved through enforcing suitable compatibility conditions of acoustic pressure and axial velocity across the geometrical discontinuities. Bibliography tends to favour the point collocation technique and mode matching method as techniques to enforce these conditions [4, 11-13]. In general, for geometries with small dimensions, mode matching has been shown to have some advantages in terms of speed and accuracy, due in part to symmetry properties, orthogonality of the transversal modes, the sensitivity of point collocation to the grid chosen and acoustic scattering at particular locations $[4,12$, 13].

On the other hand, granular absorbent materials are studied here, from an acoustical point of view, as a potential alternative to the traditional fibrous materials used in dissipative silencers. As shown in earlier studies, sound propagation in absorbent materials can be modelled through complex and frequency dependent density and speed of sound [14]. Following the same 
approach for granular materials, their acoustic properties can be predicted through models available in the bibliography [15-17], which will be used in the context of the current investigation.

In this work, a mathematical approach based on a numerical version of the mode matching method $[8,9]$ is presented to compute the transmission loss of silencers with granular material. Multidimensional sound propagation is taken into account in configurations with arbitrary, but axially uniform, cross section. Transversal material heterogeneities are included in the model $[3,11]$. Also, the possibility of using different filling levels of granular material gives rise to cross sections with an abrupt change of properties located not only in the commonly used central perforated passage, but also in the transition between air and material, that involves a remarkable change in porosity (see interface $\Gamma_{a_{-} g r}$ in Fig. 1). The computational requirements of a full numerical scheme such as FEM are reduced through a method that combines analytical axial propagation terms with numerical transversal eigensolutions of the silencer heterogeneous cross section. Numerical mode matching $[8,9]$ is then used to couple the modal expansions associated with each silencer component and to obtain the complete solution of the wave equation. To this end, the compatibility conditions of the acoustic fields (acoustic pressure and axial velocity) at the geometric discontinuities between the silencer chamber and the inlet and outlet pipes are taken into account. Transmission loss predictions show good agreement with experimental results obtained for a particular configuration. Also, the results obtained with the proposed approach are compared favourably with general three-dimensional FE computations, offering a reduction in the computational effort. Finally, a number of silencer geometries with granular material have been considered. The effect of several parameters on the acoustic attenuation has been assessed, including grain size, filling level, porosity and silencer cross section.

\section{MODE MATCHING APPROACH}

As indicated previously, the mode matching approach is combined with FEM to reduce the computation time of a full 3D FE formulation. The procedure can be 
especially useful for configurations with arbitrary, but axially uniform, cross section, which are very commonly found in commercial silencer designs. In the current study, as observed in Fig. 1, the filling level of the silencer is assumed to be uniform along the $z$-direction, according to the previous premise. In addition, several subdomains can be distinguished depending on the propagation medium: air within the central airway of the silencer, denoted by $\Omega_{A}$; granular material in the central chamber surrounding the perforated central duct, represented by $\Omega_{c g r}$, and air on the top of the chamber, described by $\Omega_{C a}$. In the context of the current work, the symbol $\Gamma$ is used to denote the boundary of a given subdomain $\Omega$, while $S$ refers to its cross section (the same subscripts associated with subdomains are also used for corresponding $\Gamma$ and $S$ to keep notation consistency). Finally, the transition air/material boundary is defined by $\Gamma_{a_{-} g r}$, whereas $\Gamma_{p}$ refers to the perforated surface used to confine the granular material inside the outer chamber of the silencer. The air is characterized by its density $\rho_{a}$ and speed of sound $c_{a}$, while the equivalent properties of the granular material are $\rho_{g r}$ and $c_{g r}$, both complex and frequency-dependent. Finally, the inlet and outlet ducts subdomains are denoted by $\Omega_{I}$ and $\Omega_{O}$.
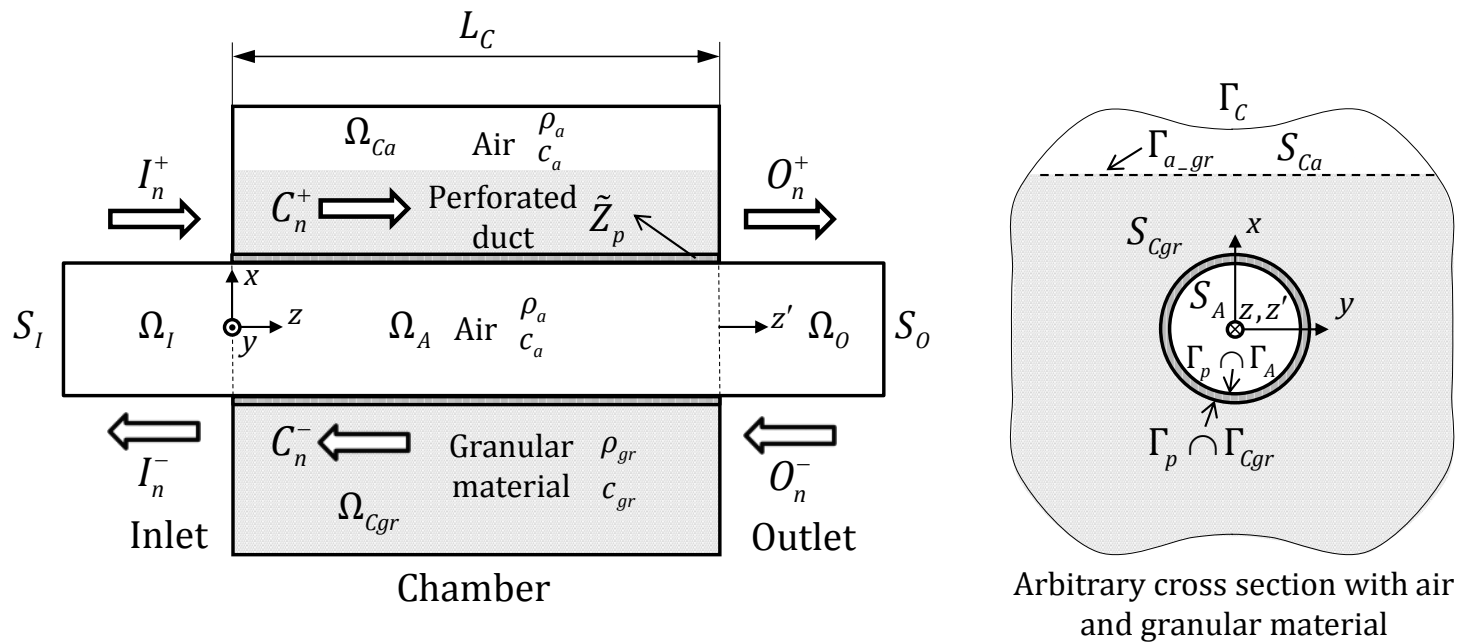

Figure 1. Scheme of silencer with granular material.

In the current investigation, only the procedure used to obtain the eigenvalues (axial wavenumbers) and eigenvectors (pressure modes) associated with the cross section of the chamber will be described, since computing the eigenvalues and eigenvectors associated with the rigid wall inlet/outlet ducts is straightforward [7, 
11, 18]. Then, the compatibility equations of the acoustic pressure and axial velocity are applied at geometric discontinuities to obtain the modal amplitudes and to compute the silencer attenuation.

\subsection{Acoustic formulation of the problem}

The sound propagation within the air subdomains $\Omega_{A}$ and $\Omega_{C a}$ is governed by the wave equation for a heterogeneous medium $[3,11]$

$$
\nabla \cdot\left(\frac{1}{\rho_{a}} \nabla P_{a}\right)+\frac{k_{a}^{2}}{\rho_{a}} P_{a}=0,
$$

where $\nabla$ is the gradient operator, $P_{a}$ the acoustic pressure, $\rho_{a}$ the air density and $k_{a}$ the wavenumber, defined as the ratio between the angular frequency $\omega$ and the speed of sound $c_{a}$. In a similar way, the wave equation in the granular material can be expressed as $[3,11]$

$$
\nabla \cdot\left(\frac{1}{\rho_{g r}} \nabla P_{g r}\right)+\frac{k_{g r}^{2}}{\rho_{g r}} P_{g r}=0,
$$

$P_{g r}$ being the acoustic pressure, $k_{g r}$ the wavenumber, and $\rho_{g r}$ the equivalent density associated with subdomain $\Omega_{C g r}$ [14]. Then, assuming a chamber with axially uniform cross section, and applying separation of variables, allows to express the acoustic pressure as $[7,8]$

$$
P(x, y, z)=\Psi^{x y}(x, y) \mathrm{e}^{-\mathrm{j} k_{z} z} \Rightarrow \Psi^{x y}(x, y)=\left\{\begin{array}{c}
\Psi_{A}^{x y}(x, y),(x, y) \in S_{A} \\
\Psi_{C a}^{x y}(x, y),(x, y) \in S_{C a} \\
\Psi_{C g r}^{x y}(x, y),(x, y) \in S_{C g r}
\end{array},\right.
$$

where $\Psi^{x y}(x, y)$ is the transversal pressure mode and $k_{z}$ the axial wavenumber. Now, combining Eq. (3) with (1) and (2) yields three tranversal wave equations for $S_{A}, S_{C a}$ and $S_{C g r}$, that can be written as

$$
\begin{aligned}
& \nabla \cdot\left(\frac{1}{\rho_{a}} \nabla \Psi_{A}^{x y}\right)+\frac{\left(k_{a}^{2}-k_{z}^{2}\right)}{\rho_{a}} \Psi_{A}^{x y}=0, \\
& \nabla \cdot\left(\frac{1}{\rho_{a}} \nabla \Psi_{C a}^{x y}\right)+\frac{\left(k_{a}^{2}-k_{z}^{2}\right)}{\rho_{a}} \Psi_{C a}^{x y}=0,
\end{aligned}
$$




$$
\nabla \cdot\left(\frac{1}{\rho_{g r}} \nabla \Psi_{C g r}^{x y}\right)+\frac{\left(k_{g r}^{2}-k_{z}^{2}\right)}{\rho_{g r}} \Psi_{C g r}^{x y}=0 .
$$

\subsection{FE formulation and quadratic eigenvalue problem}

In the framework of a 2D FE discretization, the transversal pressure $\Psi^{x y}(x, y)$ of Eq. (3) for $S_{A}, S_{C a}$ and $S_{C g r}$ can be approximated by shape functions as

$$
\Psi^{x y}(x, y)=\mathbf{N}(x, y) \Psi,
$$

where $\mathbf{N}(x, y)$ contains the nodal shape functions of each subdomain (functional dependence in terms of the independent variables $x$ and $y$ is dropped hereinafter to simplify the notation), and $\Psi$ contains the unknown nodal values of each transversal pressure mode [19]. Now, the weighted residuals method is applied to Eqs. (4)-(6) together with Green's theorem and the Galerkin approach [11, 19]. Assuming rigid wall subdomains, this leads to

$$
\begin{gathered}
\int_{S_{A}} \frac{1}{\rho_{a}}\left(\nabla \mathbf{N}^{T} \nabla \mathbf{N}+\left(k_{z}^{2}-k_{a}^{2}\right) \mathbf{N}^{T} \mathbf{N}\right) d S \Psi_{A}=\int_{\Gamma_{p} \cap \Gamma_{A}} \frac{1}{\rho_{a}} \mathbf{N}^{T} \frac{\partial \Psi}{\partial n} d \Gamma, \\
\int_{S_{C a}} \frac{1}{\rho_{a}}\left(\nabla \mathbf{N}^{T} \nabla \mathbf{N}+\left(k_{z}^{2}-k_{a}^{2}\right) \mathbf{N}^{T} \mathbf{N}\right) d S \Psi_{C a}=\int_{\Gamma_{a_{-} g r} r} \frac{1}{\rho_{a}} \mathbf{N}^{T} \frac{\partial \Psi_{C a}}{\partial n} d \Gamma, \\
\int_{S_{G g r}} \frac{1}{\rho_{g r}}\left(\nabla \mathbf{N}^{T} \nabla \mathbf{N}+\left(k_{z}^{2}-k_{g r}^{2}\right) \mathbf{N}^{T} \mathbf{N}\right) d S \Psi_{C g r} \\
=\int_{\Gamma_{a_{-} g r}} \frac{1}{\rho_{g r}} \mathbf{N}^{T} \frac{\partial \Psi_{C g r}}{\partial n} d \Gamma+\int_{\Gamma_{p} \cap \Gamma_{C g r}} \frac{1}{\rho_{g r}} \mathbf{N}^{T} \frac{\partial \Psi_{C g r}}{\partial n} d \Gamma .
\end{gathered}
$$

The coupling conditions at boundaries $\Gamma_{a_{-} g r}$ and $\Gamma_{p}$ are applied as follows. In the first case, continuity of pressure and normal acoustic flow are considered, the latter being written as

$$
\frac{1}{\rho_{a}} \frac{\partial \Psi_{C a}}{\partial n}=-\frac{\phi}{\rho_{g r}} \frac{\partial \Psi_{C g r}}{\partial n},
$$


where $\phi$ is the porosity of the granular material and $n$ represents the outward normal direction. Eq. (11) is introduced in Eq. (10), then Eq. (10) is multiplied by $\phi$ and finally Eqs. (9) and (10) are considered altogether, yielding

$$
\begin{aligned}
& \int_{S_{C a}} \frac{1}{\rho_{a}}\left(\nabla \mathbf{N}^{T} \nabla \mathbf{N}+\left(k_{z}^{2}-k_{a}^{2}\right) \mathbf{N}^{T} \mathbf{N}\right) d S \Psi_{C a} \\
& +\int_{S_{C g r}} \frac{\phi}{\rho_{g r}}\left(\nabla \mathbf{N}^{T} \nabla \mathbf{N}+\left(k_{z}^{2}-k_{g r}^{2}\right) \mathbf{N}^{T} \mathbf{N}\right) d S \Psi_{C g r}=\int_{\Gamma_{p} \cap \Gamma_{C g r}} \frac{\phi}{\rho_{g r}} \mathbf{N}^{T} \frac{\partial \Psi_{C g r}}{\partial n} d \Gamma,
\end{aligned}
$$

where it should be noted that the boundary integrals at $\Gamma_{a_{-} g r}$ have been cancelled. Eq. (12) can be written in compact form as

$$
\left(\mathbf{K}_{C}+k_{z}^{2} \mathbf{M}_{C}\right) \Psi_{C}=\int_{\Gamma_{p} \cap \Gamma_{G r} r} \frac{\phi}{\rho_{g r}} \mathbf{N}^{T} \frac{\partial \Psi_{C g r}}{\partial n} d \Gamma,
$$

where $\boldsymbol{\Psi}_{C}$ contains the unknown nodal values $\boldsymbol{\Psi}_{C a}$ and $\boldsymbol{\Psi}_{C g r}$ in $S_{C a} \cup S_{C g r}$ (the perforated central passage is not yet included). The corresponding submatrices are

$$
\begin{gathered}
\mathbf{K}_{C}=\int_{S_{C a}} \frac{1}{\rho_{a}}\left(\nabla \mathbf{N}^{T} \nabla \mathbf{N}-k_{a}^{2} \mathbf{N}^{T} \mathbf{N}\right) d S+\int_{S_{c g r}} \frac{\phi}{\rho_{g r}}\left(\nabla \mathbf{N}^{T} \nabla \mathbf{N}-k_{g r}^{2} \mathbf{N}^{T} \mathbf{N}\right) d S, \\
\mathbf{M}_{C}=\int_{S_{C a}} \frac{1}{\rho_{a}} \mathbf{N}^{T} \mathbf{N} d S+\int_{S_{c g r}} \frac{\phi}{\rho_{g r}} \mathbf{N}^{T} \mathbf{N} d S .
\end{gathered}
$$

Eq. (8) can also be written in compact form as follows

$$
\left(\mathbf{K}_{A}+k_{z}^{2} \mathbf{M}_{A}\right) \Psi_{A}=\int_{\Gamma_{p} \cap \Gamma_{A}} \frac{1}{\rho_{a}} \mathbf{N}^{T} \frac{\partial \Psi_{A}}{\partial n} d \Gamma
$$

with

$$
\begin{gathered}
\mathbf{K}_{A}=\int_{S_{A}} \frac{1}{\rho_{a}}\left(\nabla \mathbf{N}^{T} \nabla \mathbf{N}-k_{a}^{2} \mathbf{N}^{T} \mathbf{N}\right) d S, \\
\mathbf{M}_{A}=\int_{S_{A}} \frac{1}{\rho_{a}} \mathbf{N}^{T} \mathbf{N} d S .
\end{gathered}
$$

Regarding the perforated duct, the acoustic coupling of Eqs. (13) and (16) at $\Gamma_{p}$ (air in the central passage with the surrounding chamber) can be carried out by means of the perforated duct impedance. The continuity of the normal acoustic flow given by Eq. (11) is considered together with the condition corresponding to the 
impedance $\tilde{Z}_{p}$, the latter being defined as the ratio of the pressure jump to the acoustic velocity normal to surface $[1,11]$. The condition applied over $\Gamma_{\mathrm{p}}$ is expressed as follows

$$
\begin{gathered}
\frac{\partial \Psi_{A}}{\partial n}=-\rho_{a} \mathrm{j} \omega \frac{\Psi_{A}-\Psi_{C g r}}{\tilde{Z}_{p}}, \\
\frac{\partial \Psi_{C g r}}{\partial n}=\frac{\rho_{g r} \mathrm{j} \omega}{\phi} \frac{\Psi_{A}-\Psi_{C g r}}{\tilde{Z}_{p}} .
\end{gathered}
$$

After substituting Eqs. (19) and (20) in expressions (13) and (16), the following FE system of equations is obtained

$$
\begin{aligned}
& \left(\mathbf{K}_{A}+k_{z}^{2} \mathbf{M}_{A}\right) \Psi_{A}=-\int_{\Gamma_{p} \cap \Gamma_{A}} \frac{\mathbf{j} \omega}{\tilde{Z}_{p}} \mathbf{N}^{T}\left(\mathbf{N} \Psi_{A}-\mathbf{N} \Psi_{C}\right) d \Gamma, \\
& \left(\mathbf{K}_{C}+k_{z}^{2} \mathbf{M}_{C}\right) \boldsymbol{\Psi}_{C}=\int_{\Gamma_{p} \cap \Gamma_{G g r}} \frac{\mathbf{j} \omega}{\tilde{Z}_{p}} \mathbf{N}^{T}\left(\mathbf{N} \Psi_{A}-\mathbf{N} \Psi_{C}\right) d \Gamma,
\end{aligned}
$$

which is expressed in matrizant form as

$$
\left(\mathbf{K}+k_{z}^{2} \mathbf{M}\right) \mathbf{\Psi}=\mathbf{0} .
$$

The following global matrices $\mathbf{K}$ and $\mathbf{M}$ have been defined

$$
\begin{gathered}
\mathbf{K}=\left[\begin{array}{ll}
\mathbf{K}_{A_{-} A} & \mathbf{K}_{A_{-} C} \\
\mathbf{K}_{C_{-} A} & \mathbf{K}_{C_{-} C}
\end{array}\right], \\
\mathbf{M}=\left[\begin{array}{cc}
\mathbf{M}_{A} & \mathbf{0} \\
\mathbf{0} & \mathbf{M}_{C}
\end{array}\right],
\end{gathered}
$$

and all the unknown nodal values are included in vector $\Psi$. The corresponding submatrices are

$$
\begin{gathered}
\mathbf{K}_{A_{-} A}=\mathbf{K}_{A}+\int_{\Gamma_{p} \cap \Gamma_{A}} \frac{\mathbf{j} \omega}{\tilde{Z}_{p}} \mathbf{N}^{T} \mathbf{N} d \Gamma, \\
\mathbf{K}_{C_{-} C}=\mathbf{K}_{C}+\int_{\Gamma_{p} \cap \Gamma_{G g r}} \frac{j \omega}{\tilde{Z}_{p}} \mathbf{N}^{T} \mathbf{N} d \Gamma, \\
\mathbf{K}_{A_{-} C}=-\int_{\Gamma_{p} \cap \Gamma_{A}} \frac{\mathbf{j} \omega}{\tilde{Z}_{p}} \mathbf{N}^{T} \mathbf{N} d \Gamma,
\end{gathered}
$$




$$
\mathbf{K}_{C_{-} A}=-\int_{\Gamma_{p} \cap \Gamma_{G r}} \frac{\mathbf{j} \omega}{\tilde{Z}_{p}} \mathbf{N}^{T} \mathbf{N} d \Gamma .
$$

Finally, the system of equations (23) can be conveniently arranged as an eigenvalue problem $[7,11]$

$$
\left(\begin{array}{cc}
\mathbf{0} & \mathbf{I} \\
-\mathbf{M}^{-1} \mathbf{K} & \mathbf{0}
\end{array}\right)\left\{\begin{array}{c}
\boldsymbol{\Psi} \\
k_{z} \boldsymbol{\Psi}
\end{array}\right\}=k_{z}\left\{\begin{array}{c}
\boldsymbol{\Psi} \\
k_{z} \boldsymbol{\Psi}
\end{array}\right\},
$$

I being the identity matrix and $\mathbf{0}$ a matrix composed of zeros. The solution provides the axial wavenumbers and the pressure modes associated with the cross section of the chamber.

\subsection{Continuity of the acoustic pressure and axial velocity fields. Numerical} mode matching

For the subdomains involved (inlet/outlet ducts defined by $\Omega_{I}$ and $\Omega_{0}$, respectively, as well as the chamber $\Omega_{C}=\Omega_{A} \cup \Omega_{C a} \cup \Omega_{C g r}$ ), the full acoustic pressure and axial velocity fields can be described by modal expansions depending on incident and reflected waves. In the case of the inlet duct, the following expressions can be used $[1,7,11,20-22]$

$$
\begin{gathered}
P_{I}(x, y, z)=\sum_{n=1}^{\infty}\left(I_{n}^{+} \mathrm{e}^{-\mathrm{j} k_{l, n} z}+I_{n}^{-} \mathrm{e}^{\mathrm{j} k_{I, n} z}\right) \Psi_{I, n}(x, y), \\
U_{I}(x, y, z)=\frac{1}{\rho_{a} \omega} \sum_{n=1}^{\infty}\left(I_{n}^{+} \mathrm{e}^{-\mathrm{j} k_{l, n} z}-I_{n}^{-} \mathrm{e}^{\mathrm{j} k_{l, n} z}\right) \Psi_{I, n}(x, y),
\end{gathered}
$$

$I_{n}^{+}$and $I_{n}^{-}$being the unknown modal amplitudes associated with the incident and reflected waves, respectively, and $\Psi_{I, n}(x, y)$ the transversal pressure mode related to the cross section of the inlet duct (note that, compared to Eqs. (3)-(6), superscript $x y$ is dropped hereinafter to simplify the notation). Besides, $k_{I, n}$ represents the inlet duct axial wavenumber, $\omega$ the angular frequency and $j$ the imaginary unit. The acoustic fields within the chamber and the outlet duct can be analogously described after the appropriate modifications, the unknown modal 
amplitudes being $C_{n}^{+}, C_{n}^{-}, O_{n}^{+}$and $O_{n}^{-}$. From a practical point of view, the expansions are truncated to $N_{I}, N_{C}$ and $N_{O}$ terms, respectively.

To take advantage of the modal orthogonality properties, and with a view to reducing the computational effort of numerical integrations, the pressure expansion of Eq. (31) for the chamber takes into account the transversal pressure modes $\Psi_{C, n}(x, y)$, while the velocity expansion of Eq. (32) considers the mode $\Psi_{C, n, U}(x, y)=\left(\rho_{a} / \tilde{\rho}\right) \Psi_{C, n}(x, y)$. Here, $\tilde{\rho}$ is chosen depending on the subdomain of the chamber cross section involved $\left(S_{A}, S_{C a}\right.$ or $S_{C g r}$, see Fig. 1 for details). Additional details of the procedure can be found in references $[21,22]$.

With a view to computing the sound propagation within the silencer, it is necessary to evaluate all the unknown modal amplitudes. The acoustic fields are matched at geometric discontinuities to generate a suitable system of equations. Compatibility conditions are provided by continuity of acoustic pressure and axial velocity at the expansion/contraction, and zero axial velocity normal to the rigid endplates $[1,7-9,11]$. For the expansion, these equations can be written as

$$
\begin{gathered}
P_{I}(x, y, z=0)=P_{C}(x, y, z=0) \quad(x, y) \in S_{I} \equiv S_{A}, \\
U_{I}(x, y, z=0)=U_{C}(x, y, z=0) \quad(x, y) \in S_{I} \equiv S_{A}, \\
U_{C}(x, y, z=0)=0 \quad(x, y) \in S_{C}-S_{A},
\end{gathered}
$$

where sections $S_{I} \equiv S_{A}$ belong to the inlet and the central perforated passage, respectively, and $S_{C}=S_{A} \cup S_{C a} \cup S_{C g r}$ is related to the complete section of the chamber.

On the other hand, at the contraction, the compatibility equations can be written as

$$
\begin{gathered}
P_{C}\left(x, y, z=L_{C}\right)=P_{O}\left(x, y, z^{\prime}=0\right) \quad(x, y) \in S_{A} \equiv S_{O}, \\
U_{C}\left(x, y, z=L_{C}\right)=U_{O}\left(x, y, z^{\prime}=0\right) \quad(x, y) \in S_{A} \equiv S_{O}, \\
U_{C}\left(x, y, z=L_{C}\right)=0 \quad(x, y) \in S_{C}-S_{A},
\end{gathered}
$$

$S_{O}$ being the cross section of the outlet duct. 
Now, regarding the expansion, Eq. (33) is multiplied by the weighting function $\Psi_{I, m}(x, y)$ corresponding to the transversal mode of the inlet duct, and integrated over $S_{I} \equiv S_{A}$. This leads, for $m=1,2, \ldots, N_{I}$, to the following expression

$$
\int_{S_{I}} P_{I}(x, y, 0) \Psi_{I, m}(x, y) d S=\int_{S_{A}} P_{C}(x, y, 0) \Psi_{I, m}(x, y) d S .
$$

It should be noticed that, due to the orthogonality of the transversal modes, the previous equation can be simplified and written as

$$
\left(I_{m}^{+}+I_{m}^{-}\right) \int_{S_{I}} \Psi_{I, m}^{2}(x, y) d S=\sum_{n=1}^{N_{C}}\left(C_{n}^{+}+C_{n}^{-}\right) \int_{S_{A}} \Psi_{C, n}(x, y) \Psi_{I, m}(x, y) d S
$$

where no modal summation appears on the left hand side. Besides, Eqs. (34) and (35), related to the axial acoustic velocity field, can be multiplied by the incident transversal mode of the chamber $\Psi_{C, m}(x, y)$, with $m=1,2, \ldots, N_{C}$. The first equation is integrated over $S_{I} \equiv S_{A}$, and the second one over $S_{C}-S_{A}$, resulting after summation in

$$
\int_{S_{I}} U_{I}(x, y, 0) \Psi_{C, m}(x, y) d S=\int_{S_{C}} U_{C}(x, y, 0) \Psi_{C, m}(x, y) d S .
$$

Considering the orthogonality properties of the transversal modes leads to [21, 22]

$$
\begin{aligned}
\sum_{n=1}^{N_{I}} k_{I, n}\left(I_{n}^{+}-I_{n}^{-}\right) \int_{S_{I}} & \Psi_{I, n}(x, y) \Psi_{C, m}(x, y) d S \\
& =k_{C, m}\left(C_{m}^{+}-C_{m}^{-}\right) \int_{S_{C}} \Psi_{C, m, U}(x, y) \Psi_{C, m}(x, y) d S .
\end{aligned}
$$

Now, following a similar procedure for the contraction, Eqs. (36)-(38) can be rewritten as

$$
\begin{array}{r}
\sum_{n=1}^{N_{C}}\left(C_{n}^{+} \mathrm{e}^{-\mathrm{j} k_{C, n} L_{C}}+C_{n}^{-} \mathrm{e}^{\mathrm{j} k_{C, n} L_{C}}\right) \int_{S_{A}} \Psi_{C, n}(x, y) \Psi_{O, m}(x, y) d S \\
=\left(O_{m}^{+}+O_{m}^{-}\right) \int_{S_{O}} \Psi_{O, m}^{2}(x, y) d S, \\
k_{C, m}\left(C_{m}^{+} \mathrm{e}^{-\mathrm{j} k_{C, m} L_{C}}-C_{m}^{-} \mathrm{e}^{\mathrm{j} k_{C, m} L_{C}}\right) \int_{S_{C}} \Psi_{C, m, U}(x, y) \Psi_{C, m}(x, y) d S \\
=\sum_{n=1}^{N_{O}} k_{O, n}\left(O_{n}^{+}-O_{n}^{-}\right) \int_{S_{O}} \Psi_{O, n}(x, y) \Psi_{C, m}(x, y) d S,
\end{array}
$$


where Eqs. (43) and (44) are related to the acoustic pressure and axial velocity fields, respectively. The former has been multiplied by the weighting function $\Psi_{O, m}(x, y)$, with $m=1,2, \ldots, N_{O}$, and the latter by $\Psi_{C, m}(x, y)$, with $m=1,2, \ldots, N_{C}$.

Finally, the weighting integrals can be numerically computed, and the algebraic system defined by Eqs. (40), (42)-(44) provides $N_{I}+2 N_{C}+N_{O}$ equations to obtain the modal amplitudes. To compute the transmission loss $(T L)$, two additional conditions are required given by an incident plane wave at the inlet duct ( $\left.I_{0}^{+}=1, I_{n}^{+}=0, \forall n\right)$ and an anechoic termination at the outlet $\operatorname{duct}\left(O_{n}^{-}=0, \forall n\right)[7$, 8-13]. Finally, once the modal amplitudes are known, the attenuation of the silencer can be obtained as

$$
T L=-20 \log \left(\frac{S_{O}}{S_{I}}\left|O_{0}^{+}\right|\right)
$$

assuming that the outlet duct is long enough to guarantee the rapid decay of higher order evanescent modes [11, 20, 21].

\section{ACOUSTIC MODEL FOR THE GRANULAR MATERIAL}

The granular material can be modelled by means of its equivalent acoustic properties, e.g. density and bulk modulus, both complex and frequency dependent. According to the bibliography $[15,16]$, the equivalent density of the granular material can be expressed as

$$
\rho_{g r}=\rho_{a} q\left(1+\frac{\mu \sigma}{j \omega \rho_{a} k_{p} q} \sqrt{1+\frac{j \omega \rho_{a} 4 q^{2} k_{p}^{2}}{\mu \Lambda^{2} \sigma^{2}}}\right),
$$

where $q$ is the tortuosity [14], $\mu$ is the dynamic viscosity, $\sigma$ the volume porosity (which can differ from the surface porosity $\phi$ at the interfaces $\Gamma_{a_{-} g r}$ and $\Gamma_{p}$ defined in the previous section), $k_{p}$ the permeability and $\Lambda$ the viscous characteristic length defined as follows

$$
\Lambda=\frac{4(1-\Theta) \sigma q R_{\text {part }}}{9(1-\Theta)}
$$


$R_{\text {part }}$ being the particle radius (spheres in the context of the present investigation), while tortuosity is given by

$$
q=1+\frac{1-\sigma}{2 \sigma}
$$

In addition, permeability is represented by $k_{p}=\mu / R$, where $R$ is the flow resistivity that can be written as

$$
R=\frac{9 \mu(1-\sigma)}{2 R_{\text {part }}^{2} \phi^{2}} \frac{5(1-\Theta)}{5-9 \sqrt[3]{\Theta}+5 \Theta-\Theta^{2}}
$$

In Eqs. (47) and (49), $\Theta$ is the cell radius defined as

$$
\Theta=\frac{3}{\pi \sqrt{2}}(1-\sigma)
$$

In addition, the complex bulk modulus can be expressed as [16]

$$
K_{g r}=\frac{\gamma P_{0}}{\gamma-\frac{\gamma-1}{1+\frac{8 \mu}{j \omega \rho_{a} \operatorname{Pr} \Lambda^{\prime 2}} \sqrt{1+\frac{j \omega \rho_{a} \operatorname{Pr} \Lambda^{\prime 2}}{16 \mu}}}}
$$

$\gamma$ being the specific heat ratio, $P_{0}$ the atmospheric pressure, $\operatorname{Pr}$ the Prandtl number $\left(\operatorname{Pr}=C_{p} \mu / \kappa\right.$, where $C_{p}$ is the heat capacity at a constant pressure and $\kappa$ the thermal conductivity), and $\Lambda^{\prime}$ the thermal characteristic length that can be written as

$$
\Lambda^{\prime}=\frac{3 \Lambda}{2 q(1-\Theta)}
$$

Once $\rho_{g r}$ and $K_{g r}$ are obtained, the equivalent characteristic impedance of the granular material can be determined by [16]

$$
Z_{g r}=\sqrt{K_{g r} \rho_{g r}},
$$

while the wavenumber $k_{g r}$ and the speed of sound $c_{g r}$ are defined through the following expressions

$$
k_{g r}=\omega \sqrt{\frac{\rho_{g r}}{K_{g r}}},
$$




$$
c_{g r}=\frac{\omega}{k_{g r}}=\sqrt{\frac{K_{g r}}{\rho_{g r}}} .
$$

\section{RESULTS}

First, the validation of the numerical mode matching approach previously described and a study of convergence of the solution are presented. In addition, some results are shown to assess the influence of a number of parameters related to the granular material (composed of rigid spherical particles), such as the grain size, the volume of the chamber filled with spheres and the porosity. Finally, the impact of the cross section geometry has been also analysed to evaluate the acoustic behaviour of different silencer configurations with granular material. Regarding the porosity of the perforated duct, it is assumed high enough so that, in practical terms, its acoustic impedance can be neglected [1]. Therefore, its influence is not considered in any of the cases under study, detailed in Table 1.

\subsection{Validation and study of convergence}

\subsubsection{Validation}

The first silencer configuration under study presents a circular cross section, its main dimensions being: $L_{I}=L_{O}=0.1 \mathrm{~m}$ (length of the inlet/outlet ducts), $L_{C}=0.3 \mathrm{~m}$ (length of the chamber), $R_{I}=R_{O}=0.0268 \mathrm{~m}$ (radius of the inlet/outlet ducts) and $R_{C}$ $=0.091875 \mathrm{~m}$ (radius of the chamber). The air properties have been measured at room temperature $\left(21.4^{\circ} \mathrm{C}\right)$, the speed of sound being $c_{0}=344.13 \mathrm{~m} / \mathrm{s}$ and the density $\rho_{0}=1.1979 \mathrm{~kg} / \mathrm{m}^{3}$. The validation of the mode matching approach has been carried out for cases I and II (see Table 1 and, Figs. 2 and 3, respectively). In both of them the granular material presents a porosity of $39.9 \%$ and is composed of spheres of $0.006 \mathrm{~m}$ in diameter. 


\begin{tabular}{|c|c|c|c|c|}
\hline Case & $\begin{array}{c}\text { Granular } \\
\text { diameter (m) }\end{array}$ & $\begin{array}{c}\text { Volume filling of the } \\
\text { chamber (\%) }\end{array}$ & $\begin{array}{l}\text { Chamber cross } \\
\text { section shape }\end{array}$ & Porosity (\%) \\
\hline I & 0.006 & 25 & Circular & 39.9 \\
\hline II & 0.006 & 37.5 & Circular & 39.9 \\
\hline III & 0.001 & 100 & Circular & 39.9 \\
\hline IV & 0.003 & 100 & Circular & 39.9 \\
\hline V & 0.006 & 100 & Circular & 39.9 \\
\hline VI & 0.002 & 100 & Circular & 39.9 \\
\hline VII & 0.004 & 100 & Circular & 39.9 \\
\hline VIII & 0.001 & 25 & Circular & 39.9 \\
\hline IX & 0.001 & 50 & Circular & 39.9 \\
\hline $\mathrm{X}$ & 0.001 & 75 & Circular & 39.9 \\
\hline XI & 0.001 & 100 & Circular & 42 \\
\hline XII & 0.001 & 100 & Circular & 47.6 \\
\hline XIII & 0.006 & 100 & Circular & 42 \\
\hline XIV & 0.006 & 100 & Circular & 47.6 \\
\hline $\mathrm{XV}$ & 0.001 & 100 & Elliptical & 39.9 \\
\hline XVI & 0.001 & 100 & Triangular & 39.9 \\
\hline
\end{tabular}

Table 1. Silencer configurations containing spherical granular material.

The attenuation computed by means of the mode matching approach for filling cases I and II is compared in Figs. 2 and 3 with experimental measurements [22, 23] and numerical results. The former have been obtained in the experimental facilities of the research centre, and show good agreement with the $T L$ obtained through the mode matching method, thus validating the proposed numerical technique from a practical point of view. Also, a comparison is provided with full 3D FE computations carried out with the commercial package Comsol 
Multiphysics ${ }^{\circledR}$, showing an excellent agreement with undistinguishable attenuation curves. Regarding the agreement with the experimental results, however, higher discrepancies appear in filling case II (see Fig. 2b) compared with case I. The reasons will be further studied in future research. In addition, it is worth noting that the addition of granular material within the chamber increases the $T L$ in the mid and high frequency range, as expected.
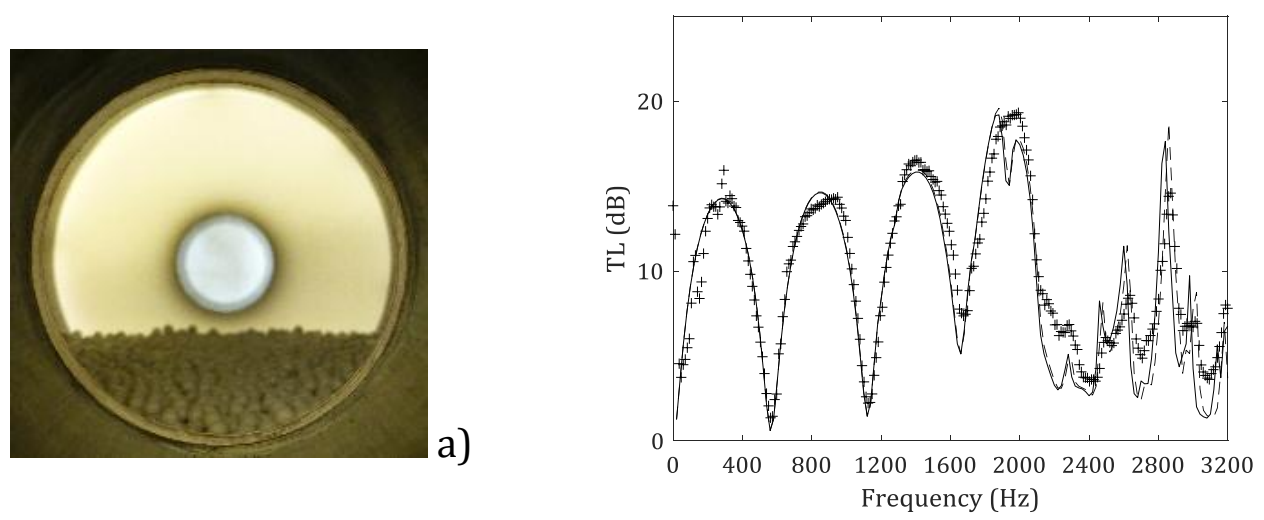

b)

Figure 2. a) Picture of the prototype; b) $T L$ of a silencer partially filled with granular material, case I: +++, experimental measurement; - , mode matching method; - - -, Comsol Multiphysics ${ }^{\circledR}$.
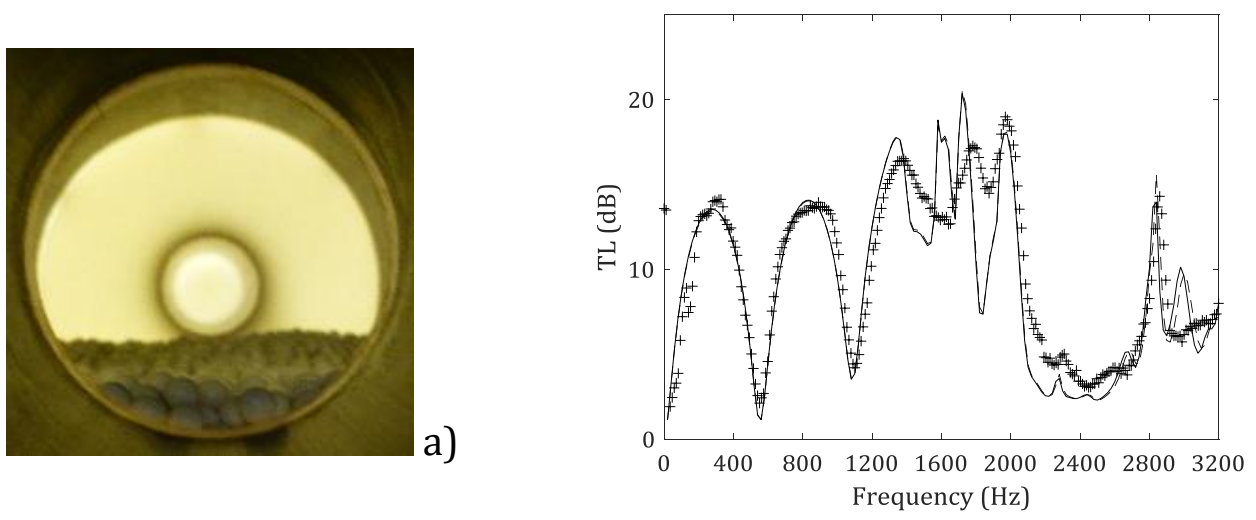

b)

Figure 3. a) Picture of the prototype; b) TL of a silencer partially filled with granular material, case II: +++, experimental measurement; - , mode matching method; - - -, Comsol Multiphysics ${ }^{\circledR}$. 


\subsubsection{Numerical impact of the weighting modes}

Several computations have been carried out to assess the accuracy and computation time of the mode matching method with an increasing number of weighting modes (see Fig. $4 \mathrm{a}$ and $4 \mathrm{~b}$ ). The geometry under consideration is the same as in the previous section, but now the chamber has been fully filled with $0.001 \mathrm{~m}, 0.003 \mathrm{~m}$ and $0.006 \mathrm{~m}$ spheres (cases III, IV and V, respectively). The transversal FE mesh of the complete chamber is composed of 104 8-node quadratic quadrilateral elements.

In order to compare the results obtained when using a different number of weighting modes, a full 3D FE "reference" solution computed with Comsol Multiphysics ${ }^{\circledR}$ has been considered. In this case, a mesh with an element size of approximately $0.001 \mathrm{~m}$ has been used to obtain accurate results. This analysis has required a computational effort of several hours due to the small size of the element compared to the dimensions of the silencer. The relative errors between the "reference" solution and the results computed with the numerical mode matching method have been computed as

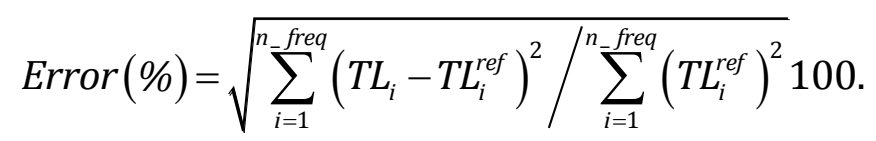

The analyses have been carried out for a frequency range from $20 \mathrm{~Hz}$ to $3200 \mathrm{~Hz}$ considering increments of $20 \mathrm{~Hz}$, and the corresponding results are shown in Fig. 4. In addition, the weighting modes considered are: 1, 2, 5, 10, 20, 40, 60, 80 and 100 for each sphere size.

As it can be observed in Fig. 4a, the relative error decreases as the number of weighting modes increases, showing the same trend for the different grain sizes. However, the condition of the problem has been measured through the reciprocal condition number. This parameter is close to zero for badly conditioned matrices and, in the current investigation, it presents values $10^{-18}, 10^{-20}$ and $10^{-21}$ for 0.001 $\mathrm{m}, 0.003 \mathrm{~m}$ and $0.006 \mathrm{~m}$ grain sizes, respectively. It can be noticed that as the diameter increases the problem tends to be worse conditioned and the error becomes higher. On the other hand, the time expenditure increases with the 
number of weighting modes (see Fig. 4b), the results being practically the same for the three cases under study, as expected. A balance between accuracy and computational cost must be found when using numerical mode matching. For example, the error for 20 modes is below $5 \%$ in all the cases and the computation time is around $450 \mathrm{~s}$, this value being much lower than the one required for the "reference" solution analysis.
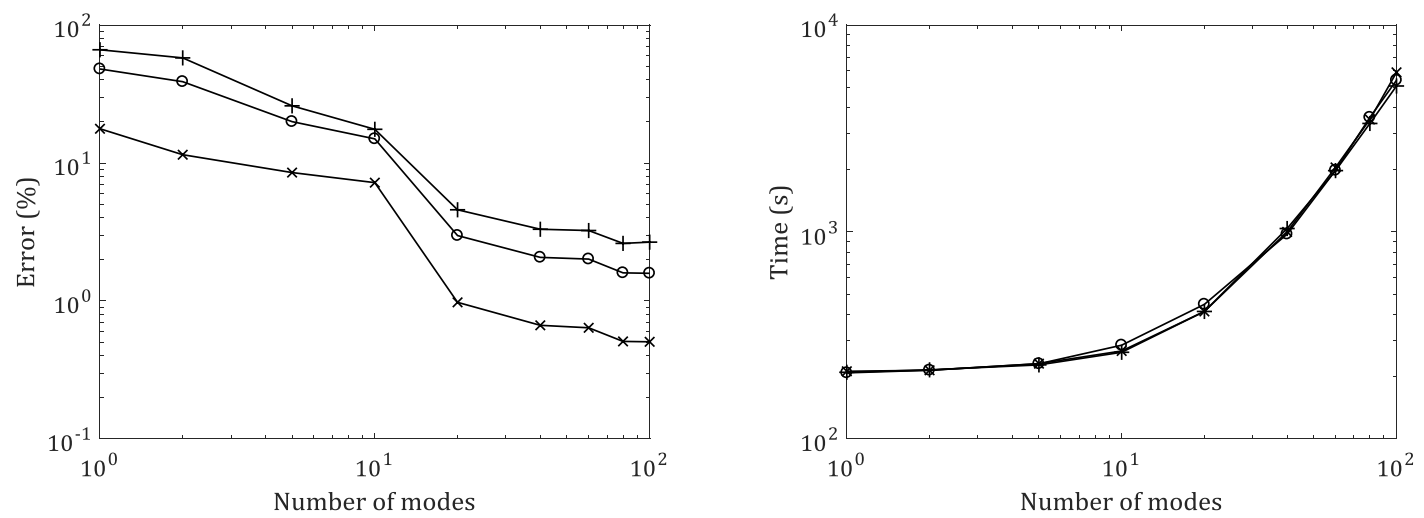

a)

b)

Figure 4. a) Relative error (\%) between the $T L$ obtained with the mode matching method and Comsol Multiphysics ${ }^{\circledR}$; b) Computation time for: xxx, case III; ooo, case IV; +++, case V.

\subsection{Influence of parameters and geometry on the attenuation}

\subsubsection{Influence of the sphere diameter on the attenuation}

The influence of the spherical granule size on the acoustic behaviour of the silencer is studied in this section. Several diameters detailed in Table 1 have been considered: $0.001 \mathrm{~m}$ (case III), $0.002 \mathrm{~m}$ (case VI), $0.004 \mathrm{~m}$ (case VII) and $0.006 \mathrm{~m}$ (case V); in all the cases the silencer outer chamber has been fully filled. As it can be observed in Fig. 5, as the sphere size decreases, the attenuation achieved increases in practically all the frequency range under consideration, in particular at mid and high frequencies. 


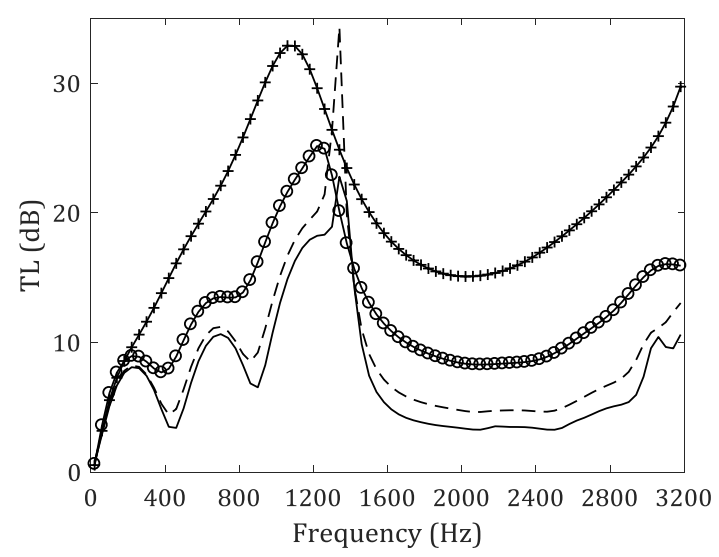

Figure 5. $T L$ of a silencer for different diameters of spherical granules: +++ , case III; - , case V; ooo, case VI; - - -, case VII.

\subsubsection{Influence of the filling level of the chamber}

The geometries considered to study the influence of the chamber filling are detailed in Table 1 and the diameter of the spherical granules is given by $0.001 \mathrm{~m}$. In the cases under study, the chamber has been filled up to $25 \%$ of the chamber volume (case VIII), 50\% (case IX), 75\% (case X) and 100\% (case III). An extra case has been also included in order to compare the acoustic behaviour of the granular material with a fibrous absorbent material, such as Owens-Corning fibre, with a resistivity of $4896 \mathrm{rayl} / \mathrm{m}$ for $100 \mathrm{~kg} / \mathrm{m}^{3}$ [21, 22]. In this latter case, a typical perforated duct has been included to confine the fibre, with hole diameter $d_{h}=$ $0.0035 \mathrm{~m}$, thickness $t=0.001 \mathrm{~m}$ and a porosity of $10 \%[1,21,22]$.

As it can be observed in Fig. 6, increasing the filling of the chamber leads to higher $T L$ in practically the whole frequency range. The behaviour of silencers with granular material tends to be similar to the configuration with fibrous material. It should be noticed that at high frequencies, and for the particular configurations under study, the attenuation achieved by some of the silencers containing granular material shows higher $T L$ values than the perforated dissipative silencer with fibres. Therefore, for some practical applications the granular material could be a potential alternative to the traditional sound absorbing fibres. 


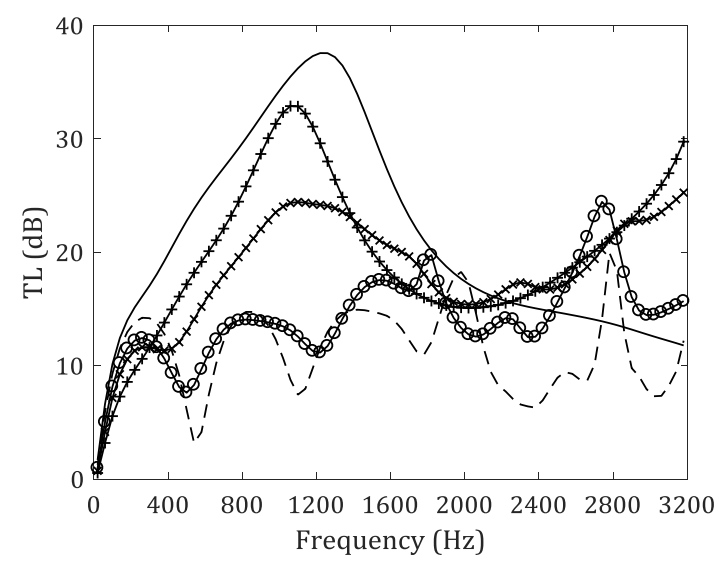

Figure 6. $T L$ of a silencer with different fillings of granular material with $0.001 \mathrm{~m}$ in diameter: +++, case III; - - -, case VIII; ooo, case IX; Xxx, case X; -, Owens-Corning fibre.

\subsubsection{Influence of porosity}

Several computations have been carried out with a view to determining the influence of the granular material porosity on the TL. Spheres can be arranged in many configurations, and the associated theoretical porosity can be calculated for each of them. If a random packing of spherical granules is assumed, porosity depends on the grain size but cannot be below 39.9\% [24]. Nevertheless, the porosity value measured by Cobo and Simón for random packings of glass beads for several diameters $(0.001 \mathrm{~m}, 0.002 \mathrm{~m}$ and $0.003 \mathrm{~m})$ was $40.5 \%$, which is close to this minimum value [17]. Therefore, a porosity value of $39.9 \%$ has been considered as the minimum porosity reachable and the maximum is assumed to be that obtained for an ideal cubic distribution, i.e. 47.6\% [24]. Two analyses have been done with porosity values of $39.9 \%$ and $47.6 \%$, as well as a third computation considering a porosity of $42 \%$. In all the cases, two different granule diameters given by $0.001 \mathrm{~m}$ and $0.006 \mathrm{~m}$ have been considered (see Table 1 ).

As it can be observed in Fig. 7, porosity seems to have more influence on the attenuation as the sphere size decreases. In fact, for grains of $0.006 \mathrm{~m}$ in diameter the effect is relatively slight and only noticeable in some narrow frequency bands. In general, lower porosities achieve higher $T L$ values with the granular material model under consideration. The effect is stronger for the smallest spheres, for 
which the influence of the porosity can be considerable (more than $5 \mathrm{~dB}$ in some frequency bands).

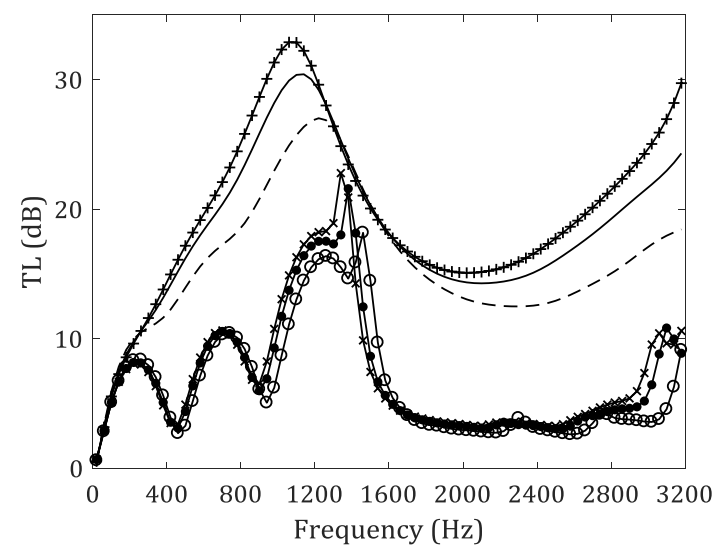

Figure 7. TL for different distribution and sizes of spheres: +++, case III; - , case XI; - - -, case XII; xxx, case V; $\cdots$, case XIII; ooo, case XIV.

\subsubsection{Transmission loss for different cross section geometries}

The attenuation delivered by several silencer configurations with different cross section (but the same area) is studied. The configurations considered are cases III, case XV and XVI, all of them defined in Table 1. In all the cases, the inlet/outlet ducts have the same circular cross section, its radius being $R_{t}=0.0268 \mathrm{~m}$. The dimensions for the concentric circular chamber (case III) have been presented in section 4.1.1, while the geometry of the elliptical configuration with offset inlet/outlet pipes (case XV) is defined by its major and minor axes as $L_{y}=0.2253 \mathrm{~m}$ and $L_{x}=0.1352 \mathrm{~m}$, the offset being $d=0.036 \mathrm{~m}$ (see Fig. 8a). Finally, the triangular cross section with rounded corners (case XVI) has $L_{y}=0.2354 \mathrm{~m}$ in height, whereas the inlet/outlet pipes are placed at a distance $d=0.1042 \mathrm{~m}$ from the bottom of the chamber. In addition, $R_{a}=0.5117 \mathrm{~m}$ is the radius of the rounded corners (see Fig. $8 b)$. In all the cases, the chamber surrounding the central duct is fully filled with spherical particles of $0.001 \mathrm{~m}$ in diameter. 

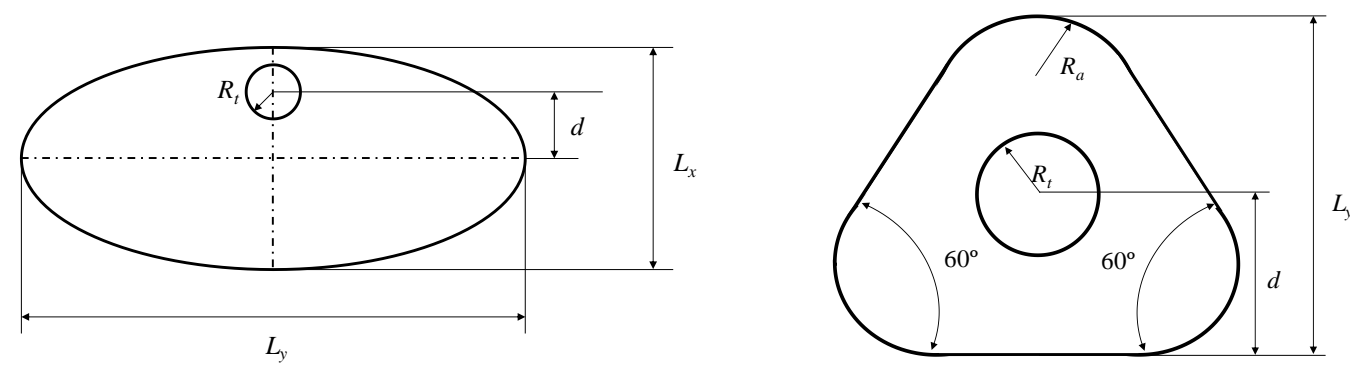

a)

b)

Figure 8. Cross section: a) elliptical chamber; b) triangular chamber with rounded corners.

Fig. 9 shows the comparison of the $T L$ corresponding to each geometry. As it can be seen, the impact of the cross section shape is significant and it is well captured by the proposed numerical methodology. The triangular configuration achieves higher attenuation values in practically the whole frequency range under study. In addition, for the particular geometries under study, the elliptical and the triangular configurations shift the attenuation peak to lower frequencies, which can be desirable for some practical silencer applications.

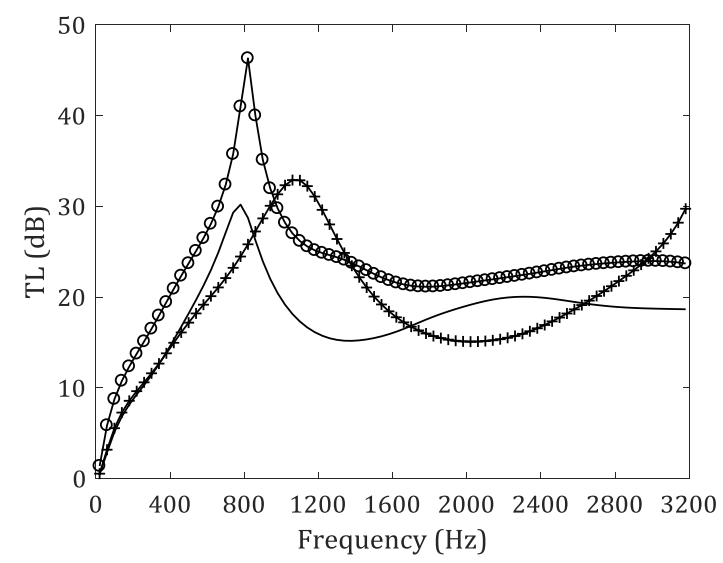

Figure 9. $T L$ of silencer with different cross section geometry: +++, case III, circular; case XV, elliptical; ooo, case XVI, triangular.

\section{CONCLUSIONS}

A numerical model based on the mode matching technique has been presented in this work to assess the acoustic behaviour of silencers with arbitrary cross section containing granular material. The approach proposed in this work has been shown to provide accurate predictions of the attenuation performance while reducing the 
computation time of a full 3D FE calculation. The model for the granular material has also been proved to be accurate enough from a practical point of view, although some discrepancies may appear as the amount of spheres within the chamber increases. In addition, decreasing the sphere size and increasing the amount of granular material lead to an improvement of the attenuation delivered by the silencer. In particular, for small granule diameters and a fully filled chamber, the silencer shows a similar acoustic performance compared to a fibrous dissipative configuration. With the material model under consideration, the silencer attenuation is higher when the granular material porosity is smaller, the latter depending on the sphere packing arrangement. Finally, the validity of the technique developed for analysing arbitrary cross sections has been proved. The corresponding geometry has been shown to have a considerable influence on the TL. Among the particular configurations under study (with the same transversal area), the triangular configuration has provided the best attenuation in almost all the frequency range.

\section{ACKNOWLEDGEMENTS}

Project supported by a 2016 BBVA Foundation Grant for Researchers and Cultural Creators. The BBVA Foundation takes no responsibility for the opinions, remarks or content included in the project and/or the results thereof, which are the sole responsibility of the authors. Support of Generalitat Valenciana (Conselleria d'Educació, Investigació, Cultura i Esport) through project Prometeo/2016/007 is also acknowledged.

\section{REFERENCES}

[1] M. L. Munjal. Acoustics of Ducts and Mufflers, Wiley, (2014). ISBN 978-1-118-44312-5.

[2] T. W. Wu. Boundary Element Acoustics, WIT Press, (2000). ISBN 1-85312-570-9.

[3] F. D. Denia, E. M. Sánchez-Orgaz, J. Martínez-Casas, R. Kirby. Finite element based acoustic analysis of dissipative silencers with high temperature and thermal-induced heterogeneity, Finite Elements in Analysis and Design, 101 (2015), 46-57.

[4] R. J. Astley. FE mode-matching schemes for the exterior Helmholtz problem and their relationship to the FE-DtN approach, Communications in Numerical Methods in Engineering, 12 (1996), 257-267. 
[5] R. Glav. The point-matching method on dissipative silencers of arbitrary cross-section, Journal of Sound and Vibration, 189 (1996), 123-135.

[6] R. Glav. The transfer matrix for a dissipative silencer of arbitrary cross-section, Journal of Sound and Vibration, 236 (2000), 575-594.

[7] R. Kirby. Transmission loss predictions for dissipative silencers of arbitrary cross section in the presence of mean flow, Journal of the Acoustical Society of America, 114 (2003), 200-209.

[8] R. Kirby. A comparison between analytic and numerical methods for modelling automotive dissipative silencers with mean flow, Journal of Sound and Vibration, 325 (2009), 565-582.

[9] Z. Fang, Z. L. Ji. Numerical mode matching approach for acoustic attenuation predictions of double-chamber perforated tube dissipative silencers with mean flow, Journal of Computational Acoustics, 22 (2014), article number 1450004.

[10] L. Yang, Z. L. Ji, T. W. Wu. Transmission loss prediction of silencers by using combined boundary element method and point collocation approach, Engineering Analysis with Boundary Elements, 61 (2015), 265-273.

[11] F. D. Denia, E. M. Sánchez-Orgaz, L. Baeza, R. Kirby. Point collocation scheme in silencers with temperature gradient and mean flow, Journal of Computational and Applied Mathematics, 291 (2016), 127-141.

[12] R. Kirby. Modeling sound propagation in acoustic waveguides using a hybrid numerical method, Journal of the Acoustical Society of America, 124 (2008), 1930-1940.

[13] F. D. Denia, J. Martínez-Casas, J. Carballeira, E. Nadal, F. J. Fuenmayor. Computational performance of analytical methods for the acoustic modelling of automotive exhaust devices incorporating monoliths, Journal of Computational and Applied Mathematics, 330 (2017), 995-1006.

[14] J. F. Allard, N. Atalla. Propagation of Sound in Porous Media: Modelling of Sound Absorbing Materials, Wiley, (2009). ISBN 978-0-47074-661-5.

[15] O. Umnova, K. Attenborough, K. M. Li. A cell model for the acoustical properties of packing of spheres, Acta Acustica united with Acustica, 87 (2001), 226-235.

[16] J. Li, S. Zhao, K. Ishibara. Study on acoustical properties of sintered bronze porous material for transient exhaust noise of pneumatic system, Journal of Sound and Vibration, 332 (2013), 2721-2734.

[17] P. Cobo, F. Simón. A comparison of impedance models for the inverse estimation of the non-acoustical parameters of granular absorbers, Applied Acoustics, 104 (2016), 119126.

[18] R. Kirby, J. B. Lawrie. A point collocation approach to modelling large dissipative silencers, Journal of Sound and Vibration, 286 (2005), 313-339.

[19] O. C. Zienkiewicz and R. L. Taylor. The finite element method. Volume I. Elsevier Butterworth-Heinemann, (2000). ISBN 978-0-75065-160-8.

[20] A. Selamet, Z. L. Ji. Acoustic attenuation performance of circular expansion chambers with offset inlet/outlet: I. Analytical approach, Journal of Sound and Vibration, 213 (1998), 601-617.

[21] A. Selamet, M. B. Xu, I. J. Lee, N. T. Huff. Analytical approach for sound attenuation in perforated dissipative silencers with inlet/outlet extensions, Journal of the Acoustical Society of America, 117 (2005), 2078-2089. 
[22] F. D. Denia, A. Selamet, F. J. Fuenmayor, R. Kirby. Acoustic attenuation performance of perforated dissipative mufflers with empty inlet/outlet extensions, Journal of Sound and Vibration, 302 (2007), 1000-1017.

[23] F. Payri, A. Broatch, J. M. Salavert, D. Moreno. Acoustic response of fibrous absorbent materials to impulsive transient excitations, Journal of Sound and Vibration, 329 (2010), 880-892.

[24] P. Glover, Petrophysics MSc Course Notes, MSc Lecture Notes, University of Leeds. 

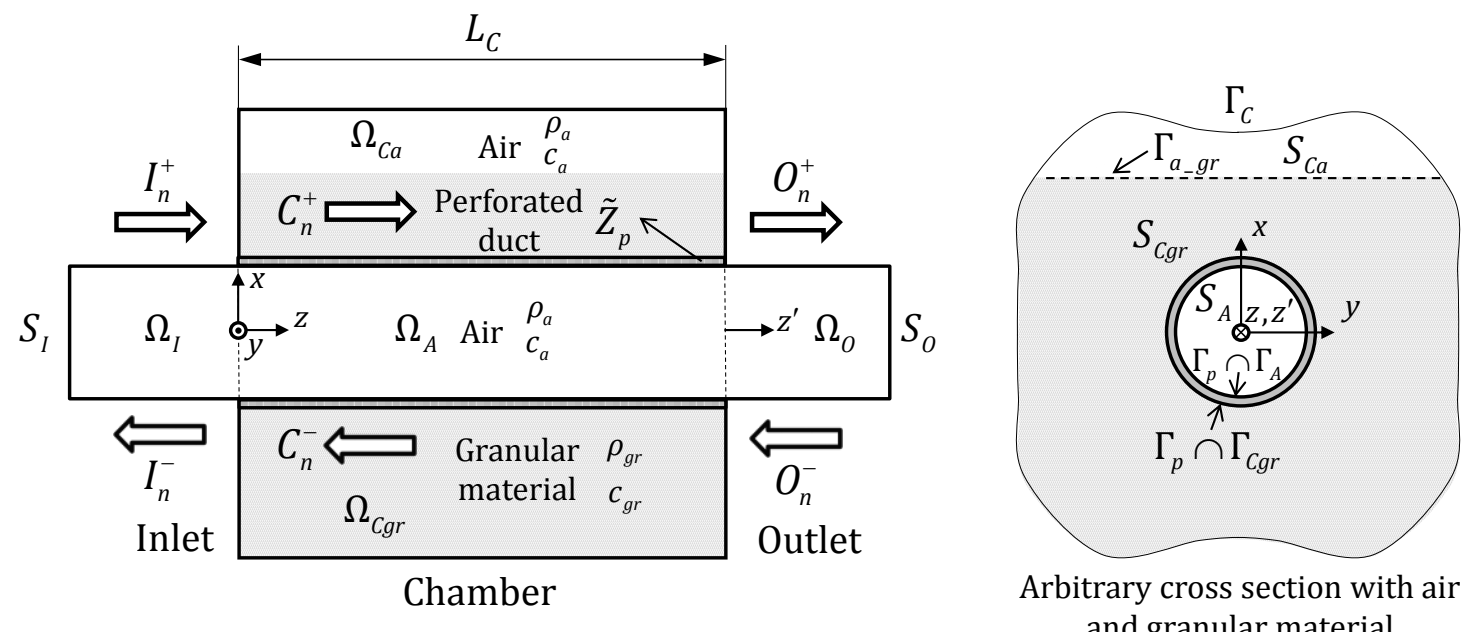

Figure 1. Scheme of silencer with granular material. 

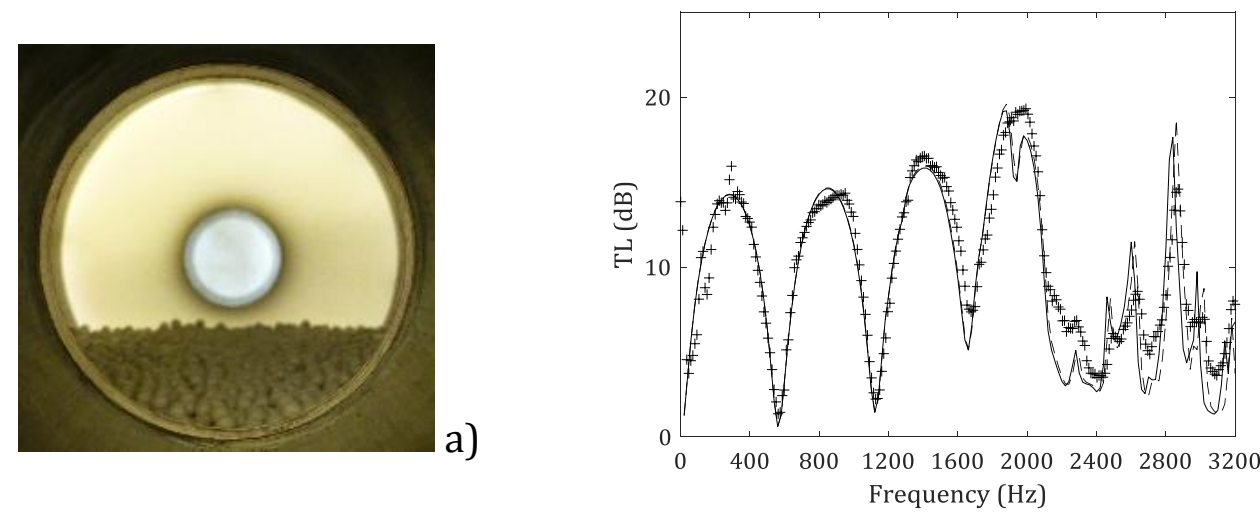

b)

Figure 2. a) Picture of the prototype; b) TL of a silencer partially filled with granular material, case I: +++, experimental measurement; - , mode matching method; - - -, Comsol Multiphysics ${ }^{\circledR}$. 

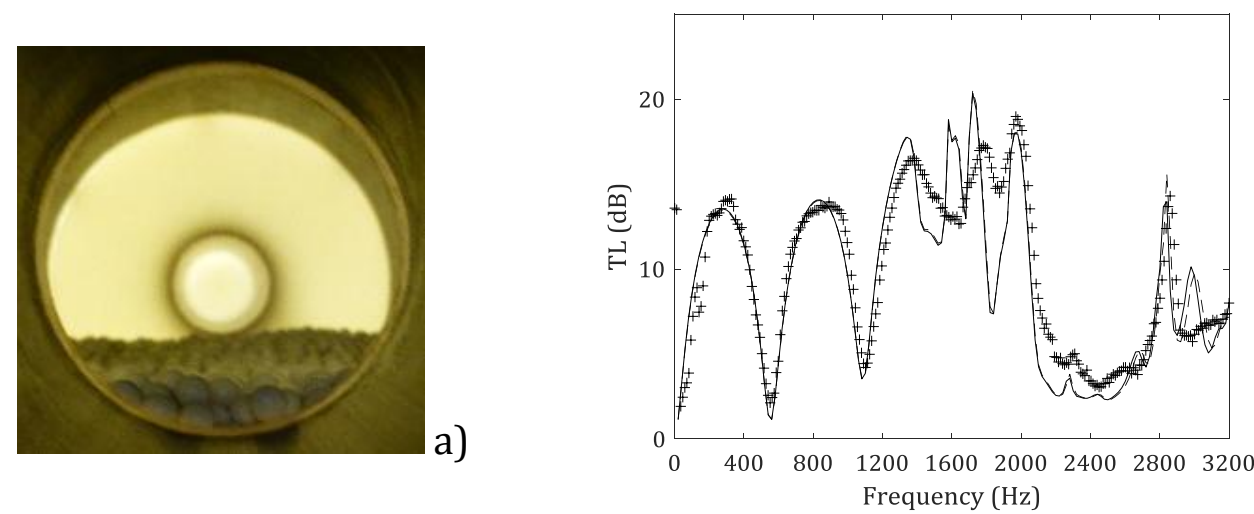

b)

Figure 3. a) Picture of the prototype; b) TL of a silencer partially filled with granular material, case II: +++, experimental measurement; - , mode matching method; - - -, Comsol Multiphysics ${ }^{\circledR}$. 

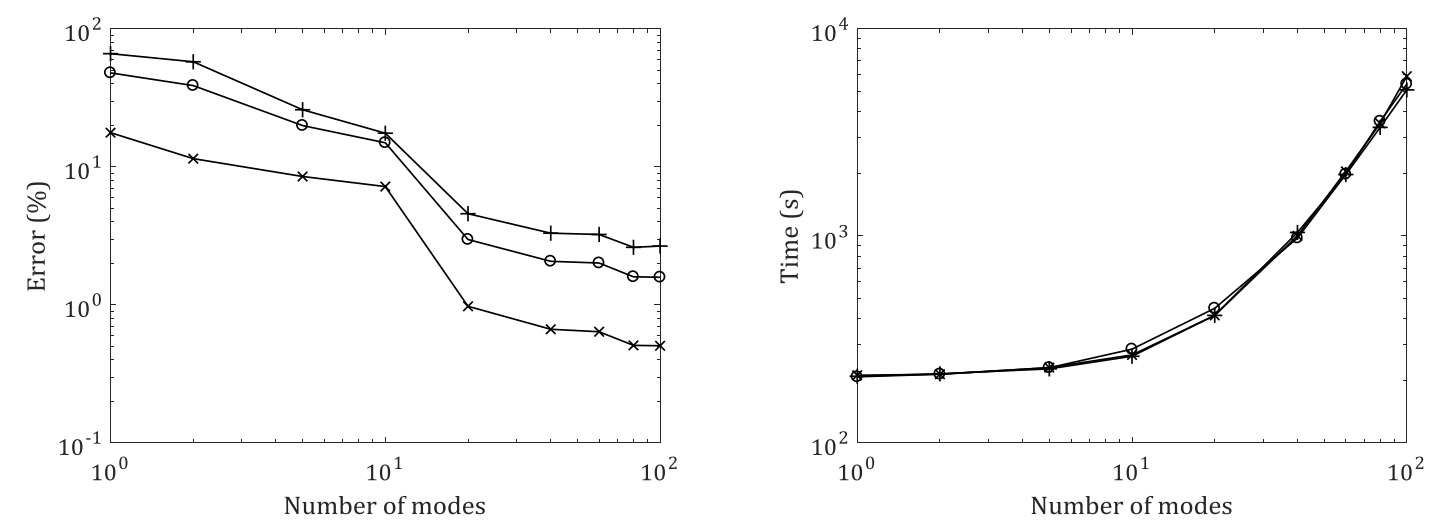

a)

b)

Figure 4. a) Relative error (\%) between the $T L$ obtained with the mode matching method and Comsol Multiphysics ${ }^{\circledR}$; b) Computation time for: xxx, case III; ooo, case IV; +++, case V. 


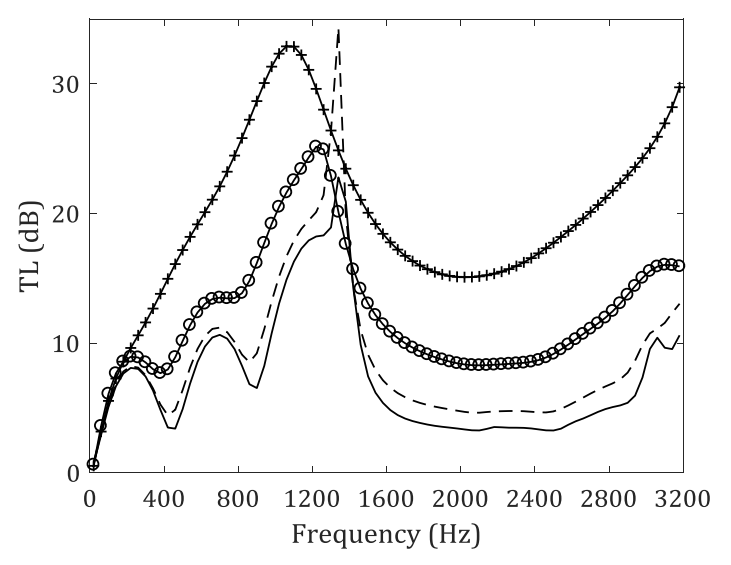

Figure 5. TL of a silencer for different diameters of spherical granules: +++, case III; -, case V; ooo, case VI; - - -, case VII. 


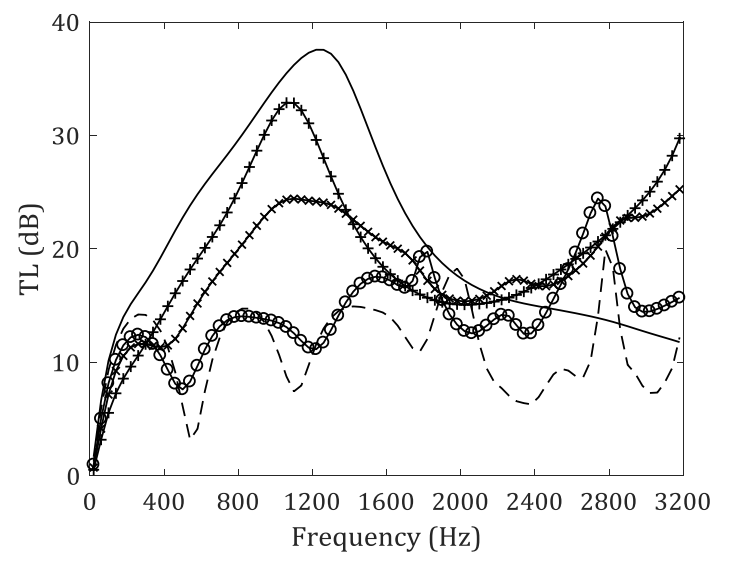

Figure 6. TL of a silencer with different fillings of granular material with $0.001 \mathrm{~m}$ in diameter: +++, case III; - - -, case VIII; ooo, case IX; xxX, case X; - , Owens-Corning fibre. 


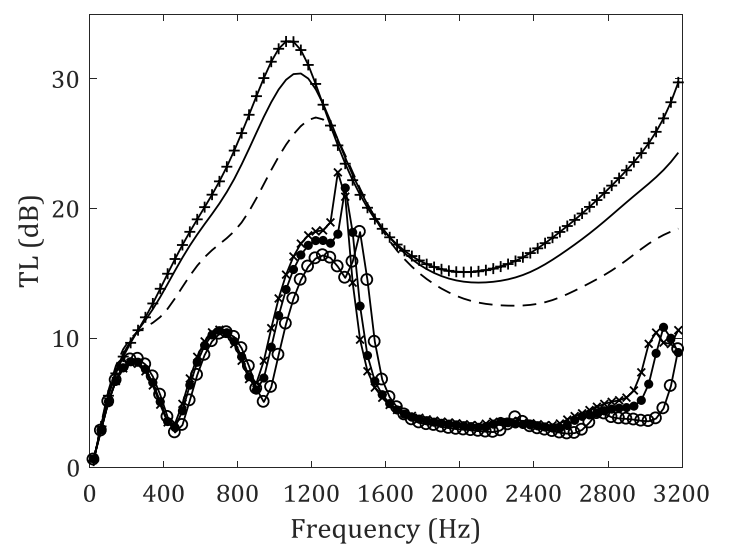

Figure 7. TL for different distribution and sizes of spheres: +++, case III; - , case XI; - - -, case XII; xxx, case V; ㄱ, case XIII; ooo, case XIV. 

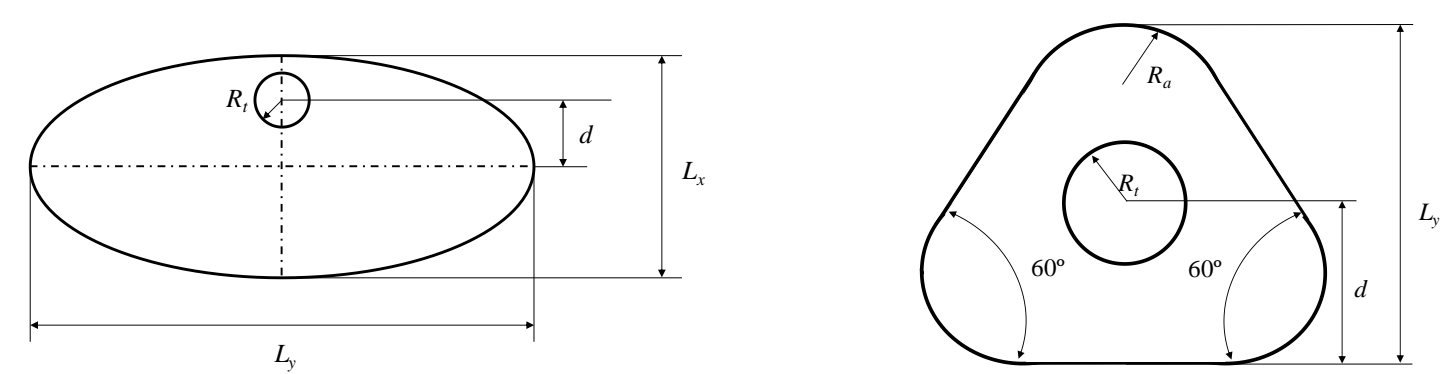

b)

Figure 8. Cross section: a) elliptical chamber; b) triangular chamber with rounded corners. 


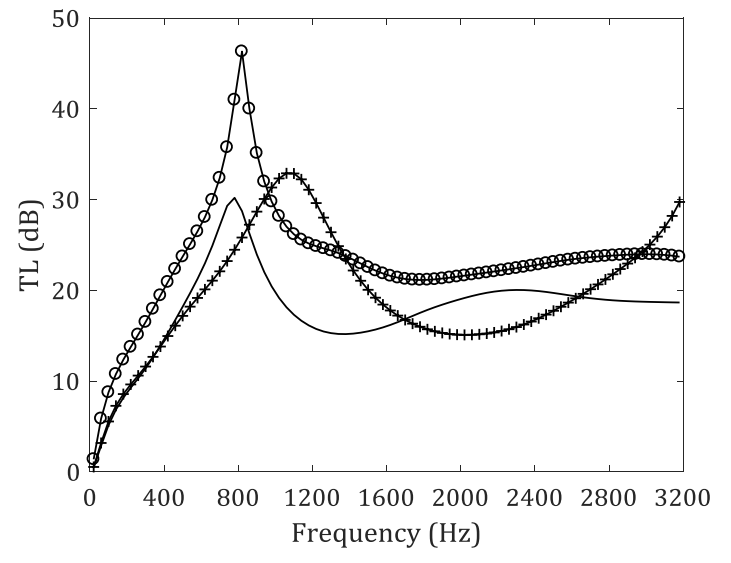

Figure 9. $T L$ of silencer with different cross section geometry: +++, case III, circular; -, case XV, elliptical; ooo, case XVI, triangular. 


\begin{tabular}{ccccc}
\hline Case & $\begin{array}{c}\text { Granular } \\
\text { diameter }(\mathrm{m})\end{array}$ & $\begin{array}{c}\text { Volume filling of the } \\
\text { chamber }(\%)\end{array}$ & $\begin{array}{c}\text { Chamber cross } \\
\text { section shape }\end{array}$ & Porosity (\%) \\
\hline I & 0.006 & 25 & Circular & 39.9 \\
II & 0.006 & 37.5 & Circular & 39.9 \\
III & 0.001 & 100 & Circular & 39.9 \\
IV & 0.003 & 100 & Circular & 39.9 \\
V & 0.006 & 100 & Circular & 39.9 \\
VI & 0.002 & 100 & Circular & 39.9 \\
VII & 0.004 & 100 & Circular & 39.9 \\
VIII & 0.001 & 25 & Circular & 39.9 \\
IX & 0.001 & 50 & Circular & 39.9 \\
X & 0.001 & 75 & Circular & 39.9 \\
XI & 0.001 & 100 & Circular & 42 \\
XII & 0.001 & 100 & Circular & 47.6 \\
XIII & 0.006 & 100 & Circular & 42 \\
XIV & 0.006 & 100 & Circular & 47.6 \\
XV & 0.001 & 0.001 & Elliptical & 39.9 \\
XVI & 0.00 & 39.9 \\
\hline & & 100 & & 4 \\
\hline
\end{tabular}

Table 1. Silencer configurations containing spherical granular material. 